\title{
The Effect of Breathing Training on the Physical Function and Psychological Problems in Patients with Chronic Stroke
}

\author{
Dong-Kyu Lee \\ Department of Physical Therapy, Sunhan Hospital, Gwangju, Korea
}

Purpose: To identify the effect of breathing training on the physical function and psychological problems in patients with chronic stroke. Methods: In total, 26 patients with chronic stroke were randomly assigned to one of two groups: the experimental group, who underwent breathing training and neurodevelopmental treatment, and the control group, who underwent neurodevelopmental treatment (13 patients per group). Physical function was evaluated using the balance and Activities of Daily Living (ADL). Psychological problems were assessed using the Depression. Balance was measured using the Berg Balance Scale (BBS). ADLs were measured using the Modified Barthel Index (MBI). Depression was measured using the Beck Depression Inventory (BDI). The BBS, MBI, and BDI were recorded before and after the 4-week treatment.

Results: As a result of comparison within groups, the experimental and control group showed significant difference for BBS, MBI and $\mathrm{BDI}$ after the experiment $(\mathrm{p}<0.05)$. In comparison between two groups, the experimental group showed more significant difference in $\mathrm{BBS}, \mathrm{MBI}$ and $\mathrm{BDI}$ than the control group $(\mathrm{p}<0.05)$.

Conclusion: This study showed that breathing training was effective improvement physical function, and psychological problems in patients with chronic stroke.

Keywords: Breathing training, Balance, ADL, Depression

\section{INTRODUCTION}

Stroke is a major adult disease and a socioeconomic burden. ${ }^{1}$ Stroke occurs when vessels supplying blood to the brain are damaged or blocked, resulting in disorders of movement, sensation, perception, cognition, and language, depending on the area damaged. ${ }^{2}$ Neurological loss is determined by the occurrence area, which may stimulate psychological disorders of anger and depression as well as physical function disorders such as sensory, exercise, balance, and walking ability body. ${ }^{3}$ Stroke may cause posture instability due to weakening of muscular strength and anesthesia, making it difficult to maintain balance. The loss of balance limits daily activities and the gait of stroke patients. ${ }^{3,4}$ Representative disorders caused by stroke include poor balance and Activities of Daily Living (ADL). ${ }^{5}$

Depression may occur in cases without a history mental illness

Received May 13, 2020 Revised Jun 15, 2020

Accepted Jun 16, 2020

Corresponding author Dong-Kyu Lee

E-mail Idkpt@hanmail.net and is a common psychological problem among stroke patients. ${ }^{3}$ It is a complication that may occur either acutely or during recovery. ${ }^{6}$ Physical function recovery becomes delayed in patients who have depression, which decreases their quality of life., ${ }^{3,6}$ Therefore, stroke patients must be comprehensively treated for both physical function disorders and psychological problems.

Stroke patients experience limited breathing function because their diaphragm, intercostal muscles, and abdominal muscle are weakened. ${ }^{7}$ Weakened breathing functions consequently decrease the ability to perform physical functions and to walk independently. ${ }^{8}$

Recently, there have been many studies on improving physical function through breathing training. ${ }^{910}$ Breathing training aims to restore the normal position and functions of diaphragm, decrease apnea, decrease breathing rate, improve movement chest wall, ensure stable airway during breathing, and increase exercise perfor-

Copylight $(02020$ The Korean Society of Physical Therapy

This is an Open Access article distribute under the terms of the Creative Commons Attribution Non-commercial License (https:// creativecommons.org/license/by-nc/4.o.) which permits unrestricted non-commercial use, distribution, and reproduction in any medium, provided the original work is properly cited. 
mance. ${ }^{11}$ The results of introducing a respiratory exercise in patients with chronic obstructive pulmonary disease showed improvement in their endurance and quality of life. ${ }^{12}$ An exercise therapy using a pneumatometer was effective in improving the pulmonary function in patients with amyotrophic lateral sclerosis. ${ }^{13}$ When breathing exercises were performed by patients with chronic obstructive pulmonary disease, the muscular strength of the diaphragm and its activity during respiratory exercises improved. ${ }^{14}$

Several studies have been conducted to date to show the effects of breathing training on patients with respiratory diseases. ${ }^{9,10}$ However, only few studies have reported the effects of breathing training, by using breathing exercise equipment, on the physical function and psychological problems in patients with chronic stroke. This study aims to identify the effects of breathing training on the physical function and psychological problems in patients with chronic stroke.

\section{METHODS}

\section{Subjects}

This study was performed on 26 patients diagnosed with stroke through CT and MRI more than 6 months ago, and randomly assigned, 13 subjects each, to the experimental (6 males, 7 females) and control (6 males, 7 females) groups. All subjects selected could perform assignments as they received a score of 24 or more on the Mini-Mental State Examination (MMSE), could walk $10 \mathrm{~m}$ or more independently, and had no visual disorder, visual field defect, and lower limb orthopedic disorder. This experiment was conducted after fully explaining the purpose of the study and the entire process of the experiment to the subjects and obtaining their consent. The study also included a signed consent form, according to the ethical standards of the Declaration of Helsinki. The general characteristics of the participants are presented in Table 1.

\section{Interventions}

The experimental and control groups received a neurodevelopmental treatment for 30 minutes a day, 5 times a week, for 4 weeks. In addition, the experimental group underwent breathing training using breathing training equipment (Lung Boost Respiratory Trainer MD8000) for 20 minutes a day, 5 times a week, for 4 weeks. Before the exercise, the patients were educated on the respiratory exercise method. They were instructed not to breathe through their nose during the respiratory exercise, and to take rest until they calmed down if they felt fatigued or dizzy, and then resume the exercise. The patient stood with both feet apart at shoulder width, sat down with the feet resting flat on the floor in a proper posture, put the respiratory exercise equipment on a head height held the equipment on one hand, pulled the chin, and made a neutral posture. The patient held a mouthpiece over the mouth, stared at the respiratory exercise equipment, and carried out inhalation and exhalation. Difficulty level 1 without resistance was applied in the first week followed by difficulty level 2 with 50\% resistance in the second week, difficulty level 3 with $60 \%$ resistance in the third week, and difficulty level 4 with $70 \%$ resistance in the fourth week (Figure 1).

\section{Experimental methods}

Physical function was evaluated using the balance and ADL. Psychological problems were assessed using the depression.

Balance was measured using the Berg Balance Scale (BBS). The BBS consists of sitting, standing, and posture change, with a total of

Table 1. General characteristics of subjects

\begin{tabular}{|c|c|c|c|}
\hline Variables (units) & $E G(n=13)$ & $C G(n=13)$ & $p$ \\
\hline Gender (male/female) & $6 / 7$ & $6 / 7$ & \\
\hline Infarction & 7 & 8 & \\
\hline Hemorrhage & 6 & 5 & \\
\hline Side of lesion (Rt/Lt) & $5 / 8$ & $7 / 6$ & \\
\hline Age (yr) & $61.14 \pm 7.86$ & $57.14 \pm 8.41$ & 0.376 \\
\hline Height (cm) & $166.01 \pm 5.44$ & $167.71 \pm 6.94$ & 0.617 \\
\hline Weight (kg) & $63.28 \pm 6.67$ & $62.01 \pm 9.14$ & 0.769 \\
\hline MMSE-K (score) & $26.57 \pm 0.97$ & $27.14 \pm 0.89$ & 0.277 \\
\hline Duration after onset (month) & $14.42 \pm 1.71$ & $13.57 \pm 1.98$ & 0.405 \\
\hline
\end{tabular}

Values are presented as mean \pm standard deviation.

EG: experimental group, CG: control group, MMSE-K: mini mental state examination-Korea. 
14 items. The total possible score is 56 , the higher the score, the better the individual's balance. ADLs were measured using the Modified Barthel Index (MBI). The MBI has a total score of 100 points

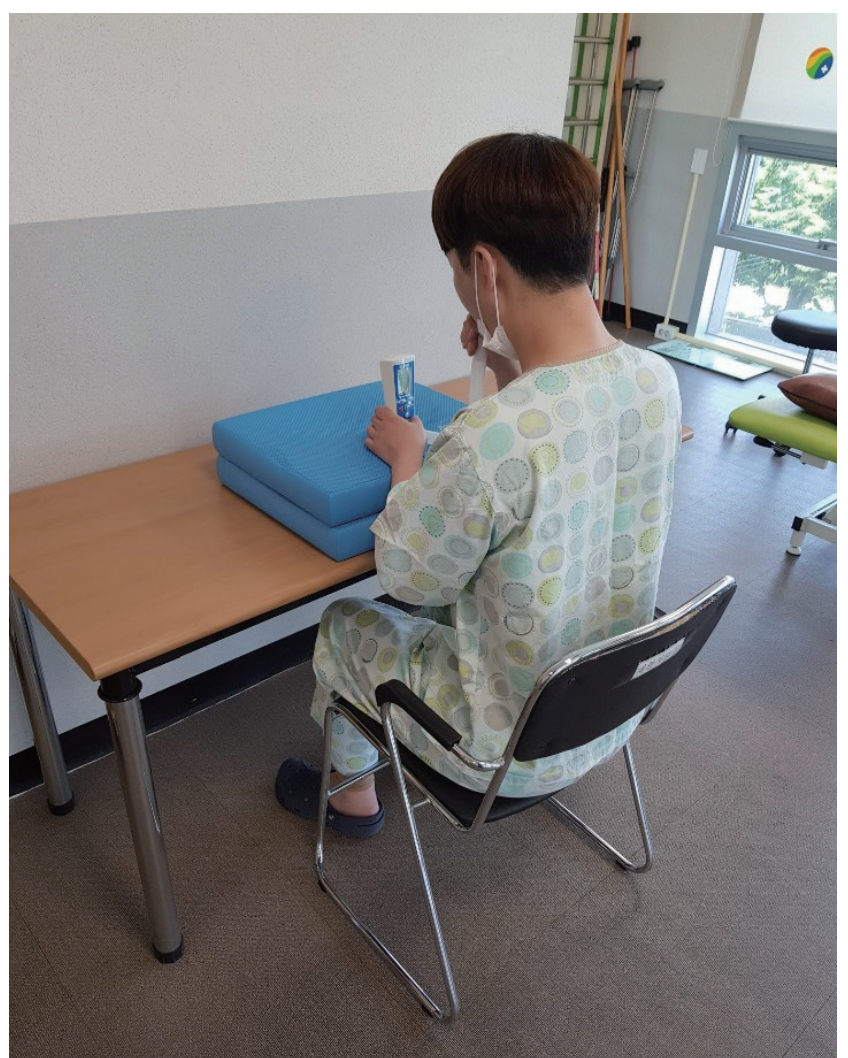

and contains a total of 10 items including personal sanitation, independent bathing, eating, using the bathroom, dressing, controlling bowel movements, controlling urination, walking or using a wheel chair, and moving towards a bed or chair. Scores of 0 to 24 indicate total dependency, scores of 25 to 49 indicate mostly dependent, 50 to 74 indicate moderate dependency, score of 75 to 90 indicate minor dependency, and score of 91 to 99 indicate minimal dependency. Depression was measured using the Beck Depression Inventory (BDI). The BDI is a four-point measurement method including 21 questions. Its total scores range from 0 to 63,9 points, $10-15$ points, 16-23 points, and $24-63$ points equating with no depression, mild depression, depression, and serious depression, respectively.

\section{Statistical analysis}

Data were analyzed using SPSS 19.0 (SPSS, Chicago, IL, USA). General features of the participants were detailed as means and standard deviations using descriptive statistics. The Shapiro-Wilk test was used to check the normal distribution of the data. The paired t-test was used to compare values before and after the experiment. An independent t-test was conducted to compare intergroup differences among changes before and after the experiment. The statistical significance level was set at $\alpha=0.05$.

Figure 1. Breathing training.

Table 2. Comparison of pre-post BBS, MBI and BDI between experimental and control group

\begin{tabular}{|c|c|c|c|c|c|}
\hline Variables & & $E G(n=13)$ & $C G(n=13)$ & $t$ & $p$ \\
\hline \multirow[t]{5}{*}{ BBS } & Pre & $39.57 \pm 1.39$ & $41.42 \pm 1.39$ & & \\
\hline & Post & $44.28 \pm 1.88$ & $43.14 \pm 1.57$ & & \\
\hline & Difference & $4.71 \pm 2.36$ & $1.71 \pm 1.49$ & 2.840 & $0.015^{*}$ \\
\hline & $\mathrm{t}$ & -5.284 & -3.032 & & \\
\hline & $p$ & $0.002^{*}$ & $0.023^{*}$ & & \\
\hline \multirow[t]{5}{*}{ MBI } & Pre & $78.14 \pm 6.91$ & $77.28 \pm 4.89$ & & \\
\hline & Post & $88.71 \pm 4.64$ & $81.42 \pm 4.35$ & & \\
\hline & Difference & $10.57 \pm 6.45$ & $4.14 \pm 3.05$ & 2.231 & $0.045^{*}$ \\
\hline & $\mathrm{t}$ & -4.335 & -2.700 & & \\
\hline & $p$ & $0.005^{*}$ & $0.036^{*}$ & & \\
\hline \multirow[t]{5}{*}{$\mathrm{BDI}$} & Pre & $21.71 \pm 0.95$ & $20.28 \pm 1.60$ & & \\
\hline & Post & $15.14 \pm 0.89$ & $18.14 \pm 1.57$ & & \\
\hline & Difference & $-6.57 \pm 0.78$ & $-2.14 \pm 1.77$ & -6.041 & $<0.001^{*}$ \\
\hline & $\mathrm{t}$ & 22.098 & 3.198 & & \\
\hline & $p$ & $<0.001^{*}$ & $0.019^{*}$ & & \\
\hline
\end{tabular}

Values are presented as mean \pm standard deviation.

EG: experimental group, CG: control group, BBS: Berg Balance Scale, MBI: Modified Barthel Index, BDI: Beck Depression Inventory.

${ }^{*} p<0.05$. 


\section{RESULTS}

\section{BBS}

The experimental group exhibited a significant difference as its BBS score, which increased from $39.57 \pm 1.39$ before treatment to $44.28 \pm$ 1.88 after treatment $(\mathrm{p}<0.05)$ (Table 2$)$. The control group exhibited a significant difference as its BBS score, which increased from 41.42 \pm 1.39 before treatment to $43.14 \pm 1.57$ after treatment $(\mathrm{p}<0.05)$ (Table 2). In comparison between the two groups, the experimental group in which breathing training was applied showed more significant change in BBS than the control group $(\mathrm{p}<0.05)$ (Table 2$)$.

\section{MBI}

The experimental group exhibited a significant difference as its MBI score, which increased from $78.14 \pm 6.91$ before treatment to $88.71 \pm 4.64$ after treatment $(\mathrm{p}<0.05)$ (Table 2$)$. The control group exhibited a significant difference as its MBI score, which increased from $77.28 \pm 4.89$ before treatment to $81.42 \pm 4.35$ after treatment $(\mathrm{p}<0.05)$ (Table 2). In comparison between the two groups, the experimental group in which breathing training was applied showed more significant change in MBI than the control group $(\mathrm{p}<0.05)$ (Table 2).

\section{BDI}

The experimental group exhibited a significant difference as its BDI score, which decreased from $21.71 \pm 0.95$ before treatment to $15.14 \pm$ 0.89 after treatment $(\mathrm{p}<0.05)$ (Table 2$)$. The control group exhibited a significant difference as its BDI score, which decreased from 20.28 \pm 1.60 before treatment to $18.14 \pm 1.57$ after treatment $(\mathrm{p}<0.05$ )(Table 2). In comparison between the two groups, the experimental group in which breathing training was applied showed more significant change in BDI than the control group $(\mathrm{p}<0.05)$ (Table 2$)$.

\section{DISCUSSION}

Breathing training improves both the respiratory muscle functions and the exercise ability of patients with weakened respiratory muscles. This study aims to identify the effects of breathing training on the physical function and psychological problems in patients with chronic stroke.

First, the study performed BBS to measure the patients' physical functions and compared BBS scores before and after the experiment. The result showed significant differences between the experimental group and control group, as well as in the BBS scores between the experimental group that had breathing training and the control group. Applying breathing training to stroke patients improved their static balance ability in the result. ${ }^{15}$ Kang et al. ${ }^{16}$ reported an improvement in the stroke patients' balance ability through a breathing training. Kang et al. ${ }^{16}$ study performed breathing training using expensive equipment that is difficult to operate for users. However, the breathing training equipment used in this study can be purchased at a low cost and is easy to operate for users even at home. Breathing training can help correct posture imbalance caused by the instability of trunk muscle as well as maintain the correct posture by playing a positive role in the stabilization of the spine and posture. ${ }^{1718}$ After breathing training, it was reported that there was a significant improvement in stroke patients' trunk muscle activity and trunk control ability ${ }^{19,20}$ These study results suggest that an improvement in trunk muscles and in trunk control ability are related, since muscle activity enhances through breathing exercise and trunk muscles are closely related to trunk control ability. ${ }^{21}$ Trunk control ability keeps the body in proper standing positions and plays a very important role in maintaining balance by controlling weight movement. ${ }^{21}$ The current and the previous study results demonstrate that there was a significant improvement in the balance ability as an increase in trunk muscles after breathing training affected the trunk control ability. Based on the study results, it is considered necessary to have intervention for a breathing training in stroke patients' rehabilitation given the improvement in their balance ability due to breathing training.

The results of comparing the MBI score before and after the experiment showed that there were significant differences in the experimental group and the control group. Also, there was a significant difference in the MBI score of experimental group that performed breathing training compared to the control group. Breathing training enhances ADL because it improves exercise resistance force. ${ }^{22}$ Exercise resistance force is the ability of inspiratory muscle to take air into lungs. ${ }^{22}$ Increasing exercise resistance force increases ventilation per minute and reduces the fatigue time of exercise. ${ }^{22,23}$ It has been reported that ADL improved after breathing training by stroke patients. ${ }^{24}$ Sutbeyaz et al. ${ }^{25}$ conducted a breathing training on stroke patients and the results demonstrated improvements in their 
performance of ADL and their quality of life. Breathing training enlarges the reduced lung volume and enhances the exercise resistance force, ultimately helping to improve the ADL of stroke patients. ${ }^{26}$ The loss of trunk muscles in stroke patients indicates a decrease in stability to maintain correct posture. ${ }^{20}$ However, breathing training is considered to improve the trunk muscles, which also bring positive effects on physical functions and ADL. The results of preceding studies suggest the necessity of breathing training applied in this study to improve stroke patients' ADL.

Depression is a serious complication that frequently starts among stroke patients in the early stage and even in the chronic period, negatively affects the prognosis, and acts as a barrier to treatment. ${ }^{27,28}$ To date, most of the stroke treatments have focused on the physical function aspects and neglected the psychological problems aspects. ${ }^{28}$ Thus, this study was conducted to examine the effect of breathing training on the psychological function of chronic stroke patients through BDI. The results comparing BDI scores before and after the experiment showed significant differences between the experimental group and the control group. Moreover, there was a significant difference in the BDI score of experimental group compared to the control group. The results of breathing training performed by Seo and Jung ${ }^{29}$ showed positive improvement in the psychological state of patients. In addition, Hyun et al. ${ }^{30}$ significantly improved the stroke patients' depression after applying breathing training. The results of the current and preceding studies were consistent despite the differences in subject selection. This study that an improvement in physical functions positively affects psychological functions. ${ }^{31}$ Since the physical functions of chronic stroke patients improved after applying breathing training in this study, the training is considered to have improved psychological issues. Breathing training helps improve chronic patients' physical functions and thus had positive effects on psychological problems. This study verified that applying breathing training to stroke patients improves their balance and ADL and is also effective in alleviating their depression. This study showed that breathing training was effective in improvement physical function, and psychological problems in patients with chronic stroke.

There are a few limitations to this study. First, it is difficult to generalize our results because of the small number of participants. Furthermore, we could not conduct a follow-up study to investigate the long-term the effects of breathing training after treatment termina- tion. To generalize the results of our study, it will be necessary to conduct a long-term follow-up study using a larger in patients with chronic stroke in the future.

\section{REFERENCES}

1. Feigin VL, Krishnamurthi RV, Parmar P et al. Update on the global burden of ischemic and hemorrhagic stroke in 1990-2013: the GBD 2013 study. Neuroepidemiology. 2015;45(3):161-76.

2. Hochstenbach J, Donders R, Mulder T et al. Long-term outcome after stroke: a disability-orientated approach. Int J Rehabil Res. 1996;19(3):189200.

3. Singh A, Black SE, Herrmann N et al. Functional and neuroanatomic correlations in poststroke depression: the Sunnybrook Stroke Study. Stroke. 2000;31(3):637-44.

4. Ikai T, Kamikubo T, Takehara I et al. Dynamic postural control in patients with hemiparesis. Am J Phys Med Rehabil. 2003;82(6):463-9.

5. Hendrickson J, Patterson KK, Inness EL et al. Relationship between asymmetry of quiet standing balance control and walking post-stroke. Gait Posture. 2014;39(1):177-81.

6. Sagen U, Vik TG, Moum T et al. Screening for anxiety and depression after stroke: comparison of the hospital anxiety and depression scale and the Montgomery and Asberg depression rating scale. J Psychosom Res. 2009;67(4):325-32.

7. Lanini B, Bianchi R, Romagnoli I et al. Chest wall kinematics in patients with hemiplegia. Am J Respir Crit Care Med. 2003;168(1):109-13.

8. Lord SE, McPherson K, McNaughton HK et al. Community ambulation after stroke: how important and obtainable is it and what measures appear predictive. Arch Phys Med Rehabil. 2004;85(2):234-9.

9. Kang TW, Kim BR. The effects of respiratory muscle training on respiratory function, balance, and activities of daily living in patients with stroke. J Kor Phys Ther. 2018:30(2):58-62.

10. Kang JI, Jeong DK. The effect of exhalation breathing exercise on respiratory synergist muscle activity and pulmonary functions in patients with forward head posture. J Kor Phys Ther. 2016;28(2):149-54.

11. Troosters T, Casaburi R, Gosselink R et al. Pulmonary rehabilitation in chronic obstructive pulmonary disease. Am J Respir Crit Care Med. 2005;172(1):19-38.

12. Koppers RJ, Vos PJ, Boot CR et al. Exercise performance improves in patients with COPD due to respiratory muscle endurance training. Chest. 2006;129(4):886-92.

13. Kim JS, Shin WS. The effects of respiratory muscle strengthening training on pulmonary function and gait ability in subacute stroke patients. J Korean Soc Phys Med. 2013;8(4):489-96.

14. Kim CY, Lee JS, Kim HD et al. Effects of the combination of respiratory muscle training and abdominal drawing-in maneuver on respiratory muscle activity in patients with post-stroke hemiplegia: a pilot randomized controlled trial. Top Stroke Rehabil. 2015;22(4):262-70.

15. Lee JH, Kim SH. Effects of closed chain lower limb exercise with abdomial breathing exercise on trunk control and balance in stroke patients. J Korean Acad Ther. 2018;10(2):31-7.

16. Kang JI, Kim BR, Park SK et al. Effects of deep abdominal muscle 
strengthening exercises on pulmonary function and the ability to balance in stroke patients. J Kor Phys Ther. 2015:27(4):258-63.

17. Corrêa EC, Bérzin F. Mouth Breathing Syndrome: cervical muscles recruitment during nasal inspiration before and after respiratory and postural exercises on Swiss Ball. Int J Pediatr Otorhinolaryngol. 2008;72(9): 1335-43.

18. Obayashi H, Urabe Y, Yamanaka Y et al. Effects of respiratory-muscle exercise on spinal curvature. J Sport Rehabil. 2012;21(1):63-8.

19. Kim CY, Lee JS, Kim HD et al. Effects of the combination of respiratory muscle training and abdominal drawing-in maneuver on respiratory muscle activity in patients with post-stroke hemiplegia: a pilot randomized controlled trial. Top Stroke Rehabil. 2015;22(4):262-70.

20. Kim MH, Lee WH, Yun MJ et al. The effects on respiratory strength training on respiratory function and trunk control in patient with stroke. J Kor Phys Ther. 2012:24(5):340-7.

21. Hodges PW, Gandevia SC. Activation of the human diaphragm during a repetitive postural task. J Physiol. 2000;522(1):165-75.

22. Sezer N, Ordu NK, Sutbeyaz ST et al. Cardiopulmonary and metabolic responses to maximum exercise and aerobic capacity in hemiplegic patients. Funct Neurol. 2004;19(4):233-8.

23. Gomes-Neto M, Saquetto MB, Silva C et al. Effects of respiratory muscle training on respiratory function, respiratory muscle strength, and exercise tolerance in patients poststroke: a systematic review with meta-anal- ysis. Arch Phys Med Rehabil. 2016;97(11):1994-2001.

24. Kim BR, Kang JI, Kim YN et al. Effects of respiratory muscle strengthening exercise on respiratory function and activities of daily living in stroke patients. J Kor Phys Ther. 2017:29(1):1-6.

25. Sutbeyaz ST, Koseoglu F, Inan L et al. Respiratory muscle training improves cardiopulmonary function and exercise tolerance in subjects with subacute stroke: a randomized controlled trial. Clin Rehabil. 2010; 24(3):240-50.

26. Haas A, Rusk HA, Pelosof $\mathrm{H}$ et al. Respiratory function in hemiplegic patients. Arch Phys Med Rehabil. 1967;48(4):174-9.

27. Primeau F. Post-stroke depression: a critical review of the literature. Can J Psychiatry. 1988;33(8):757-65.

28. Morris PL, Raphael B, Robinson RG. Clinical depression is associated with impaired recovery from stroke. Med J Aust. 1992;157(4):239-42.

29. Seo TH, Jung DI. The effects of breathing exercise on the lung function and psychological state of male smokers. KACPT. 2016;4(1):7-14.

30. Hyun KS, Won JS, Kim WO et al. The effects of danjeon breathing exercise on vital capacity, physical fitness, anxiety and depression among older adults. J Korean Acad Community Health Nurs. 2009;20(4):47482.

31. Lee DK, Kim EK. The influence of horseback riding training on the physical function and psychological problems of stroke patients. J Phys Ther Sci. 2015;27(9):2739-41. 